\title{
Free Vascularized Fibular Strut Autografts to the Lumbar Spine in Complex Revision Surgery: A Report of Two Cases
}

\author{
Bryan M. Saltzman', David M. Levy', Venus Vakhshori', Christopher J. DeWald ${ }^{1}$ \\ ${ }^{I}$ Department of Orthopedic Surgery, Rush University Medical Center, Chicago, IL, \\ ${ }^{2}$ University of Pennsylvania College of Medicine, Philadelphia, PA, USA
}

This case report presents two patients who underwent fibular strut grafting for complex revisions of previous lumbar spine arthrodeses. A case review of the Electronic Medical Record at the index institution was performed to evaluate the timeline of events of the two patients who underwent fibular strut grafting for complex revisions of previous lumbar spine arthrodesis, including imaging studies, progress notes, and laboratory results. One patient had developed chronic L3 vertebral body osteomyelitis from a prior fibular allograft and instrumentation placed for a traumatic burst fracture. The second patient had a severe scoliosis recalcitrant to prior arthrodeses in the context of Marfan syndrome and a persistent L4-5 pseudarthrosis. Both patients underwent free vascularized fibular autograft revision arthrodeses. At most recent long-term follow-up, both patients had improved clinically and neither had required further revision. The use of free vascularized fibular grafting is an excellent option for a variety of spinal indications, and these two reports indicate that the technology may have an indication for use after multiple failed surgeries for osteomyelitis or correction of a multi-level large spinal deformity secondary to Marfan syndrome.

Key Words: Autografts $\cdot$ Fibula $\cdot$ Lumbar vertebrae $\cdot$ Marfan syndrome $\cdot$ Revision $\cdot$ Surgical

\section{INTRODUCTION}

Free vascularized fibular bone grafts (FVFBGs) can be an important tool for spine surgeons to treat difficult conditions. FVFBG was introduced by Huntington in 1905 for partial tibial reconstruction ${ }^{19)}$ and is now used for reconstruction of segmental bone defects throughout the body. Its use has recently extended to spine surgery, such as multi-level corpectomies, failed arthrodesis, and poor bone quality due to radiation exposure or infection which inherently has a higher degree for complications $^{19,21)}$. A vascular graft transfers living bone and thus retains cellular viability, leading to less bony remodeling and better maintenance of its structural integrity ${ }^{19}$. Compared to avascular bone grafts, vascular grafts in the spine provide stronger, stiffer constructs with higher fusion rates and better

\footnotetext{
- Received: April 19, 2015 - Revised: July 1, 2015

- Accepted: August 5, 2015

Corresponding Author: Bryan M. Saltzman, MD

Department of Orthopedic Surgery, Rush University Medical Center, 1611

West Harrison Street, Suite 300, Chicago, IL 60612, USA

Tel: +847-989-9333, Fax: +312-942-2102

E-mail: bryan.m.saltzman@gmail.com

®This is an Open Access article distributed under the terms of the Creative

Commons Attribution Non-Commercial License (http://creativecommons.org/ licenses/by-nc/3.0/) which permits unrestricted non-commercial use, distribution, and reproduction in any medium, provided the original work is properly cited.
}

functional outcomes ${ }^{7,16,18)}$. Failed arthrodeses and posttraumatic deformity have responded to free fibular grafts with reliable rates of union and curvature correction ${ }^{4,6,10,12,14)}$. This technique has also allowed for more aggressive management of spinal neoplasms, including vertebral column resection $1^{18)}$.

We describe two complex revision cases utilizing a vascularized fibular strut autograft to the spine for failed lumbar spine arthrodeses. Our first case patient underwent vascularized grafting to augment his anterior stabilization in the context of chronic L 3 vertebral body osteomyelitis. The second patient had a complex history of more than ten previous arthrodesis and revision procedures for complex scoliosis secondary to Marfan syndrome.

\section{CASE REPORT}

\section{Case 1}

The first patient was a 46-year-old male with prior myocardial infarction and hypertension who was referred to the senior author's orthopedic spine clinic in February 2006. A motor vehicle accident in 1999 had resulted in an L3 vertebral burst fracture with lower extremity weakness. He underwent an anterior decompression and reconstruction of the $\mathrm{L} 3$ vertebral body with fibular strut allograft. He recovered all lower extremity motor function post-operatively, but in 2002, he 
was involved in a second motor vehicle accident and developed osteomyelitis of the L3 body shortly thereafter. In 2003, he underwent a removal of hardware and irrigation and debridement followed by repeat debridements in 2004 and late 2005 . Just two weeks prior to referral to the senior author's clinic, the patient noted a one-centimeter $(\mathrm{cm})$ region of dehiscence with serosanguinous exudate at his previously healed left lateral abdominal incision. Computed tomography (CT) imaging demonstrated bridging bone from L2 to L4 with a 2.0 by $1.5 \mathrm{~cm}$ subcutaneous fluid collection and a deep abscess anterior to the left psoas muscle measuring 1.3 by $0.8 \mathrm{~cm}$ (Fig. 1). The patient was recalcitrant to nonoperative management with IV antibiotic use; thus, he was scheduled for surgical intervention.

In May 2006, the patient underwent the first of a two-stage intervention with decompression of the anterior L3 abscess and posterior spinal fusion with bilateral pedicle screw instrumentation from L2 to L4 and iliac crest autograft. Cultures from the L3 vertebral body returned no growth, but the bone pathology showed chronic osteomyelitis. Postoperatively he received IV ertapenem until the second stage operation in August 2006, where the patient underwent the second stage anterior fusion with vascularized free fibula grafting. The patient was placed in the left lateral decubitus position and a flank incision was made just below the diaphragm for access to the retroperitoneal space. The L3 vertebral body was accessed at which time five $\mathrm{mL}$ of frank pus were expressed. The previous allograft struts were separated from the vertebral body with an osteotome and removed. This debridement left a bony defect measuring $3 \mathrm{~cm}$ in length and $2 \mathrm{~cm}$ in depth. Next, the free fibula graft was harvested with the assistance of a microscopic-surgery trained surgeon. A posterolateral approach to the distal right lower extremity was utilized. Small muscular branches about the fibula were sacrificed when harvesting the pedicle, but the remaining posterior tibial vessels and nerve were retained. The nutrient vessels entering the bone were carefully dissected. The harvested fibula was $3 \mathrm{~cm}$ in length, and the remaining fibular bone stock was used as autograft bone about the spine.

The fibular graft was impacted into the defect at the L3 level. The epigastric artery and vein attached to omentum were utilized as the recipient vessels to anastomose to the free fibular vessels. A telescoping technique was incorporated in which the fibular vessel was placed within the recipient vessel, and the anastomosis was performed under microscopic guidance with 9-0 Nylon sutures. Excellent inflow was noted into the graft. A nonvascularized fibular strut was inserted within the midportion of the vertebral body for structural support. The vascularized piece was then resized for a press fit at the corpectomy site and tapped into place.
Postoperatively, the patient received eight weeks of IV imipenem. He was able to tolerate ambulation with an assistive device as early as postoperative day number two. He remained hemodynamically stable with good pain control, and was discharged home after 10 days in the hospital. He was fitted for a thoracolumbosacral orthosis for six weeks after surgery. Over the next several months, the patient saw improvement in his lower back pain and no further drainage from his incision sites. Plain radiographs in April 2007 (Fig. 2A) and a repeat CT scan in August 2007 (Fig. 2B) demonstrated excellent healing and incorporation of the fibular autograft. He was weaned off of antibiotics entirely and has followed annually without complication or recurrence of his infection (Fig. 3).
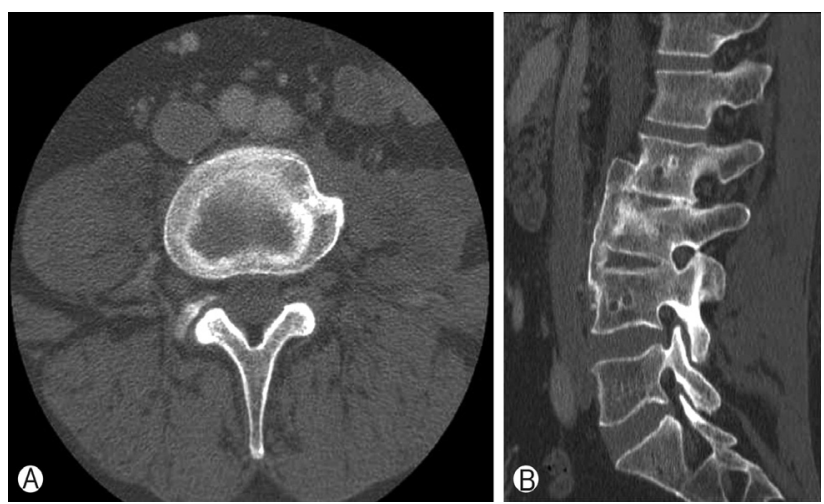

Fig. 1. Case 1. Preoperative Computed Tomography (CT) Imaging. (A) Axial CT imaging slice demonstrating a deep abscess anterior to the left psoas muscle, with (B) sagittal slice demonstrating the presence as well of bridging bone from $L 2$ to $L 4$.
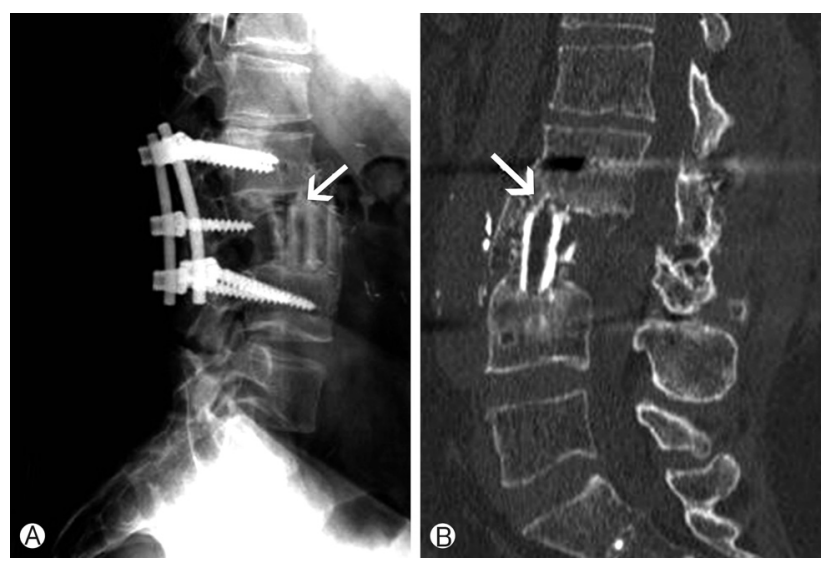

Fig. 2. Case 1. Early Follow-up Radiographic and Computed Tomography (CT) Imaging. (A) Lateral plain radiographic imaging demonstrating incorporation of the fibular autograft between the L2-L4 construct. (B) Subsequent CT imaging redemonstrates this via this sagittal cut. Note: Arrow depicts the location of the vascularized fibular autograft within the construct. 


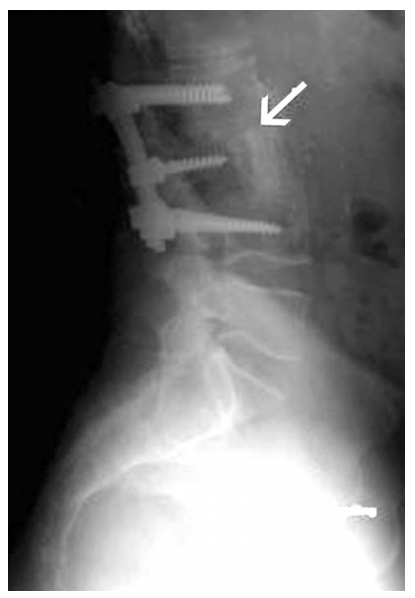

Fig. 3. Case 1. Final Followup Radiographic Imaging. Lateral plain radiographic imaging from final followup demonstrating excellent healing of the final construct. Note: Arrow depicts the location of the vascularized fibular autograft within the construct.

\section{Case 2}

The second patient is a 39-year-old female with Marfan syndrome who underwent a vascularized fibular strut autograft in 2001. The patient had endured four posterior spinal fusions before the age of 21 in an attempt to correct her severe scoliosis. She first reported to the senior author's clinic in March 1996. She had only mild lower back pain but significant difficulty breathing, swallowing, and ambulating. In standing with her knees locked, she had a 45-degree sagittal plane deformity in the thoracolumbosacral region, with severe thoracic lordosis as well as cervical and lumbosacral kyphosis. A complete reversal of the normal sagittal contours was present. In the coronal plane, she had a 35-degree levoscoliosis that corrected to 15 degrees when flexing the right lower extremity. A CT scan confirmed that she had dural ectasia, a known complication of Marfan syndrome.

In August 1996, the patient began a series of staged osteotomies and revision fusions of her spine over the following three years to address her sagittal imbalance and radiculopathies, including the following: L3-5 posterior osteotomies, sacral and pelvic posterior fixation, and removal of the inferior half of her prior Luque ring and Harrington rod constructs; anterior L4 corpectomy, L3-5 anterior spinal fusion, and harvesting of the right $8^{\text {th }}$ and $9^{\text {th }}$ cartilaginous ribs; posterior revision of her instrumented fusion from T10 to S1; right L3-5 decompression of nerve roots and removal of the right $\mathrm{L} 5$ pedicle screw and L3-4 transverse connector; revision posterior spinal fusion with removal of fractured inferior rods and iliac screw replacement.

Over the following year, the patient continued to have persistent lower back pain, decreased function, and worsening deformity. On examination, she had a significant loss of height, sagittal contour, and abdominal volume due to her lumbar spine kyphosis. In December 2000, she underwent yet another revision for Luque rod loosening and an L4-5 pseudarthrosis. The construct was revised with new left S1 pedicle and iliac screws, a left Isola rod, and transfixator proximally at the T12 level. However, her pain continued post-operatively and the deformity again progressed with persistence of the L4-5 pseudarthrosis. Her continually failing posterior hardware was attributed to a defect in the L4 region anteriorly. It was determined that a vascularized fibular strut autograft would be the most appropriate option to fill this void.

In May 2001, the patient underwent an anterior spinal fusion from L3 to sacrum with a vascularized fibular graft. A transperitoneal midline laparotomy was utilized to expose the defect at L4. The left common iliac vessels were mobilized in order to allow passage of the anastomosis. The right vascularized fibular graft was harvested and cut to the appropriate length. The fibular artery and vein were end-to-side anastomosed to the inferior mesenteric artery and vein. The L 3 vertebral body and left sacral ala were entered and spanned using the vascularized fibular graft. A 26-mm cortical screw was inserted through the construct for additional support. The remaining autograft bone was morcelized and mixed with demineralized bone matrix and packed around the vascularized fibula and excoriated bone surfaces.

Postoperatively, the patient spent several months rehabilitating in inpatient and outpatient centers. She had continued back and radicular pain and weakness, treated with therapy and chronic analgesics. She spent several weeks in an inpatient pulmonology center for co-morbidities related to her Marfan syndrome. A CT scan performed 1 year postoperatively found that the fibular strut and screw had maintained appropriate anterior stabilization. After two years, she had improved function and mobility but an unchanged level of pain and analgesic use. Plain radiographs at that time showed no interval changes or hardware failure. At 3-years postoperative, the patient's mobility had continued to progress but her level of pain persisted. She still had a large residual scoliosis and a significant thoracic lordosis, but radiographs demonstrated solid consolidation of the fibular strut. At her most recent 13-years postoperative visit, her hardware has remained intact without any signs of failure (Fig. 4).

\section{DISCUSSION}

When a decision is made to utilize graft material intraoperatively, there are several types of graft substrates available for use. From a basic overview, this includes various formations of cancellous and cortical allografts and autografts. Not uncommonly, strut grafts have been used in spinal disease or deformity 


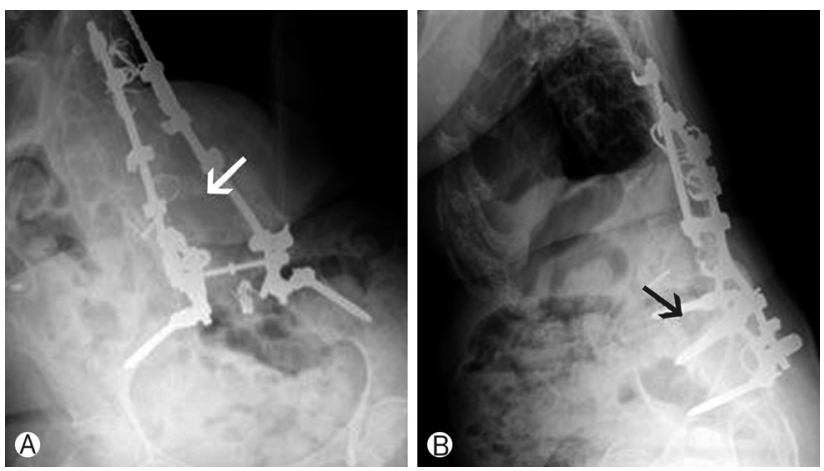

Fig. 4. Case 2. Final Follow-up Radiographic Imaging. (A) Anteroposterior and (B) lateral plain radiographic lumbar spine imaging demonstrating final follow-up with healed vascularized fibular strut and intact hardware, although with a large residual scoliosis and a significant thoracic lordosis. Note: Arrow depicts the location of the vascularized fibular autograft within the construct.

to span a potential distance and provide structural support to a construct as the fusion process is evolving. Nonvascularized grafts, including iliac crest or rib for example, are mechanically weak in the initial remodeling phase because of increased porosity of the bone, and can fatigue fracture in this time period at rates of up to $20 \%{ }^{20}$. These nonvascular graft options have inferior compressive and torsional strengths when compared with vascularized autogenous grafts (such as the fibula) which demonstrate accelerated rates of incorporation related due to its early biomechanical stability ${ }^{24)}$. Since these vascularized grafts heal similar to fracture healing in native bone, fusion likely proceeds faster than in their nonvascular counterparts and can potentially respond to external stresses similar to normal bone with reactive hypertrophy. In comparison to vascularized grafts from other anatomic locations such as the rib or iliac crest, the fibula has a particularly reliable vascular pedicle and nutrient artery in addition to a straight tubular shape to facilitate its use in the spine ${ }^{22)}$.

FVFBGs have been reported for a variety of spinal conditions including osteomyelitis, neoplasms, neurofibromatosis, and Arnold-Chiari malformations ${ }^{2,3,5,6,15)}$. Winters et al. ${ }^{23)}$ reported a case series of 30 spine patients with FVFBGs. While the authors did not present formal outcomes data, they described various techniques and an assortment of anastomosing vessels that can be used throughout the cervical, thoracic, lumbar, and sacral spine. They highlighted that complex spine cases make patients more susceptible to hypothermia, poor peripheral circulation, and thus deep vein thrombosis (DVT) of the fibular graft site. They reported peroneal DVTs in three fibular flaps, which they attributed to a prolonged period between raising the flap and transferring it to the spine. For this reason, the authors recommend keeping the donor site warm and covered for as long as possible and raising the flap as close as possible to the moment of transfer ${ }^{23)}$. Utility of the FVFBG is greatest when local tissue is compromised due to infection or radiation, for the combined periosteal and endosteal vascularity of the bone graft enhances healing. For large skeletal defects, FVFBGs demonstrate faster healing, increased structural integrity, and enhanced resistance to infection compared to nonvascular grafts ${ }^{19)}$. As opposed to other vascularized donor sites, such as the iliac crest or rib, the fibula is a long cylindrical bone with a strong cortex, reliable vascular pedicle, and little donor site morbidity ${ }^{9,13)}$.

While much of the prior literature on FVFBG use in the spine has focused on primary fusions of the cervical, thoracic, and sacral spine $e^{1,2,6-8,10,12,15,17)}$, the two cases presented in this review occurred under revision circumstances in the lumbar spine. Grafts in both patients were vascularized successfully using intra-abdominal vessels: the epigastric artery and vein in one patient and the inferior mesenteric artery and vein in the other. Both patients had clinical improvement with fusion confirmed on postoperative imaging. The first patient has had no recurrence of his spinal infection, and the second patient has had improved function and mobility without the need for further revision.

Postoperative revascularization of vascularized fibular grafts can be monitored with advanced imaging techniques including selective angiography, gadolinium-enhanced MRI, three-phase technetium-99m bone scintigraphy, single-photon emission CT, positron emission tomography, or the use of a skin paddle ${ }^{23)}$. Of these, only the use of a skin paddle has been demonstrated to be a reliable indicator of the vascularization and viability of the $\mathrm{FVFBG}^{11)}$; in the case of lumbar spinal reconstruction surgery, the inclusion of a skin paddle is not possible and as such no formal monitoring for revascularization was performed on these patients. However, their successful fusion in an appro priate time window may be considered an indicator of their viability although without formally proving so.

\section{CONCLUSION}

The use of free vascularized fibular grafting is an excellent option for a variety of spinal indications, and these two reports indicate that the technology may have an indication for use after multiple failed surgeries for osteomyelitis or correction of a multi-level large spinal deformity secondary to Marfan syndrome.

\section{REFERENCES}

1. Ackerman DB, Rose PS, Moran SL, Dekutoski MB, Bishop AT, Shin AY: The results of vascularized-free fibular grafts in complex spinal reconstruction. J Spinal Disord Tech 24:170-176, 2011 
2. Aliano KA, Agulnick M, Cohen B, Gonya G, Low C, Stavrides $S$, et al: Spinal reconstruction for osteomyelitis with free vascularized fibular grafts using intra-abdominal recipient vessels: A series of three cases. Microsurgery 33:560-566, 2013

3. Asazuma T, Yamagishi M, Nemoto K, Amako M, Osada M, Fujikawa K: Spinal fusion using a vascularized fibular bone graft for a patient with cervical kyphosis due to neurofibromatosis. J Spinal Disord 10:537-540, 1997

4. Chen ZC, Fan KF, Wu WC, Chen HC: Fusion of the lumbar spine with a free vascularized fibular bone graft: case report. Changgeng Yi Xue Za Zhi 21:463-468, 1998

5. de Kleuver M, van Jonbergen JP, Langeloo DD: Asymptomatic massive dural ectasia associated with neurofibromatosis type 1 threatening spinal column support: treatment by anterior vascularized fibula graft. J Spinal Disord Tech 17:539-542, 2004

6. Freidberg SR, Gumley GJ, Pfeifer BA, Hybels RL: Vascularized fibular graft to replace resected cervical vertebral bodies. Case report. J Neurosurg 71:283-286, 1989

7. Goldberg VM, Shaffer JW, Field G, Davy DT: Biology of vascularized bone grafts. Orthop Clin North Am 18:197-205, 1987

8. Hu H, Winters HA, Paul RM, Wuisman PI: Internal thoracic vessels used as pedicle graft for anastomosis with vascularized bone graft to reconstruct C7-T3 spinal defects: a new technique. Spine (Phila Pa 1976) 32:601-605, 2007

9. Huang YC, Leong CP, Pong YP, Liu TY, Kuo YR: Functional assessment of donor-site morbidity after harvest of a fibula chimeric flap with a sheet of soleus muscle for mandibular composite defect reconstruction. Microsurgery 32:20-25, 2012

10. Hubbard LF, Herndon JH, Buonanno AR: Free vascularized fibula transfer for stabilization of the thoracolumbar spine. A case report. Spine (Phila Pa 1976) 10:891-893, 1985

11. Imran Y, Zulmi W, Halim AS: Skin paddle as an indicator of the viability of vascularized fibular graft. Singapore Med J 45: 110-112, 2004

12. Krishnan KG, Müller A: Ventral cervical fusion at multiple levels using free vascularized double-islanded fibula - a technical report and review of the relevant literature. Eur Spine J 11:176-182,
2002

13. Ling XF, Peng X: What is the price to pay for a free fibula flap? A systematic review of donor-site morbidity following free fibula flap surgery. Plast Reconstr Surg 129:657-674, 2012

14. Minami A, Kaneda K, Satoh S, Abumi K, Kutsumi K: Free vascularised fibular strut graft for anterior spinal fusion. J Bone Joint Surg Br 79:43-47, 1997

15. Moche JA, Chopra K, Gastman B: Vascularized free fibula for cervical spine reconstruction following complicated retropharyngeal abscess. Otolaryngol Head Neck Surg 145:178-179, 2011

16. Moran SL, Bakri K, Mardini S, Shin AY, Bishop AT: The use of vascularized fibular grafts for the reconstruction of spinal and sacral defects. Microsurgery 29:393-400, 2009

17. Nijland EA, van den Berg MP, Wuisman PI, van Royen BJ, Winters HA, van Ouwerkerk WJ: Correction of a dystrophic cervicothoracic spine deformity in Recklinghausen's disease. Clin Orthop Relat Res 349:149-155, 1998

18. Sakuraba M, Kimata Y, Iida H, Beppu Y, Chuman H, Kawai A: Pelvic ring reconstruction with the double-barreled vascularized fibular free flap. Plast Reconstr Surg 116:1340-1345, 2005

19. Shin AY, Dekutoski MB: The role of vascularized bone grafts in spine surgery. Orthop Clin North Am 38:61-72, 2007

20. Streitz W, Brown J, Burnett C: Anterior fibular strut grafting in the treatment of kyphosis. Clin Ortohp Rel Res 128:140-148, 1997

21. Sung SH, Chang UK: Evaluation of risk factors for vertebral com pression fracture after stereotactic radiosurgery in spinal tumor patients. Korean J Spine 11:103-108, 2014

22. Weiland A: Current concepts review: vascularized bone autografts. J Bone Joint Surg Am 63:166-169, 1981

23. Winters HA, Kraak J, Oosterhuis JW, de Kleuver M: Spinal reconstruction with free vascularised bone grafts; approaches and selection of acceptor vessels. Scand J Surg 102:42-48, 2013

24. Wittenberg RH, Moeller J, Shea M, White AA $3^{\text {rd }}$, Hayes WC: Compressive strength of autologous and allogenous bone grafts for thoracolumbar and cervical spine fusion. Spine (Phila Pa 1976) 15:1073-1078, 1990 